\title{
Simulation of the Induced Electric Fields in the Breast Tissue During MRI Breast Imaging
}

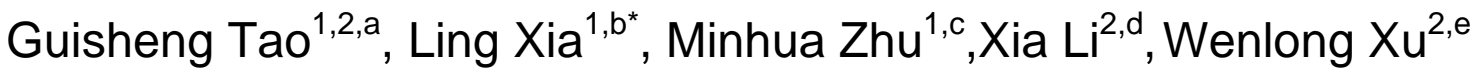 \\ ${ }^{1}$ Department of Biomedical Engineering, Zhejiang University, Hangzhou, China \\ ${ }^{2}$ Department of Information Engineering, China Jiliang University, Hangzhou, China \\ ataoguis@cjlu.edu.cn, bxialing@zju.edu.cn, czmhbme@gmail.com, lixia@cjlu.edu.cn, \\ ewenlongxu@cjlu.edu.cn
}

Keywords: breast gradient coils; induced current; quasi-static finite difference (QSFD); MRI

\begin{abstract}
This paper studies induced electric fields and current densities for peripheral nerve stimulation (PNS) induced by breast gradient coils. The numerical calculations are based on an efficient, quasi-static, finite-difference (QSFD) scheme. The application of the human body to the calculations of induced current densities and electric fields from applied low frequency magnetic and electric fields is described. The spatial distributions of the induced electric field and their gradients are calculated. This study provides some insight into the spatial characteristics of the induced field gradients for PNS in MRI, which may be used to further evaluate the sites where magnetic stimulation is likely to occur and to optimize gradient coil design.
\end{abstract}

\section{Introduction}

During MRI diagnostic imaging and spectroscopy, the patient being scanned and the individuals in the immediate vicinity of MRI equipment may be exposed to three types of magnetic and radiofrequency fields simultaneously: the high static magnetic field B0 for generating the macroscopic nuclear magnetization, the alternating magnetic gradient fields $G$ for spatial encoding the NMR signal, and the radiofrequency (RF) electromagnetic fields B1 for exciting and receiving the NMR signal [1].

Considering the safety issue in the human body during MRI scan, there are a variety of numerical approaches, including finite difference time domain (FDTD), finite element (FE) or method of moment (MOM) techniques have been developed to evaluate the induced electromagnetic field caused by the gradient field and RF field in anatomic models of the human body [2]. The FDTD method is well suited to electromagnetic analyses in MRI applications, due to its simplicity and efficiency in wave modeling and its ability to handle fields interactions and nonlinear phenomena [3, 4]. Quasi-static finite difference (QSFD) method [5], on the other hand, is ideal for linear low-frequency problems with time-harmonic dependency.

In this work, an efficient quasi-static finite difference formulation and a realistic model of an adult female Ella within realistic breast gradient coils [6] have been used. The focus of this work is on the computation of electric field and current density distributions in the breast tissue during MRI breast imaging. The electric current in the human body induced by the breast gradient coils may stimulate the peripheral nerve, and therefore this study is helpful for the optimal design of gradient coils which are with low induced current in the breast tissue.

\section{Methods}

\section{A. The Human Model}

This project is supported by the 973 National Key Basic Research \& Development Program(2010CB732500), the National Nature Science Funds of China (81101031, 30900332), Program for Science and Technology Department of Zhejiang Province (2010C14010, 2010C33172) and the natural science foundation of Zhejiang Province (Y2090966). 
The human model used in this work was a female Ella. The original spatial resolution of the model is $1 \mathrm{~mm}$ and the height of the model is $1.68 \mathrm{~m}$. With a voxel resolution of $1 \mathrm{~mm}$, the digital model has $530 * 300 * 1680$ voxels along the X, Y, Z Cartesian axes, respectively. For the computations presented here, the model is mapped onto a 5-mm grid with volume-averaged dielectric properties. The chest of the body model is embedded in the bounding box.

The experimentally determined conductivity values by Italian National Research Council [7], of some 40 body-identified tissue types were aptly scaled to the frequency of interest and assigned to the appropriate body voxels.

\section{B. Breast Gradient Coils}

The gradient coils were employed as dedicated breast gradients to evaluate the exposure of a model breast to the low-frequency magnetic fields. The breast gradient coils were from our collaborator --- the School of Information Technology and Electrical Engineering at the University of Queensland [6].

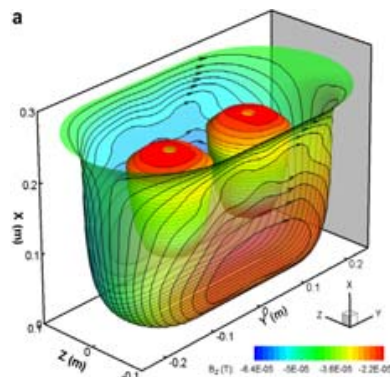

(a)

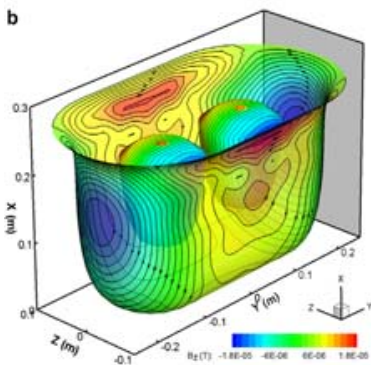

(b)

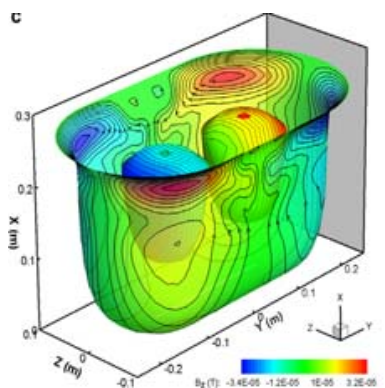

(c)

Figure. 1 The current patterns of the gradient coils. (a) x coil, (b) y coil, (c) z coil

Table1. Characteristics of the X-, Y-, and Z-gradient coil using the EMC from breast imaging

\begin{tabular}{cccc}
\hline Properties & x-Gradient & z-Gradient & y-Gradient \\
\hline Max. aperture in z,y (mm) & 280,500 & & \\
Depth in x (mm) & 260 & & \\
DSV-x-y-z (mm) & $140.5-120-120.8$ & & \\
Wire diameter (mm) & 6.6 & 2.72 & 1.7 \\
\hline
\end{tabular}

\section{The Numerical Approach}

At low source frequencies, where the dimensions of a biological body are small compared to the wavelength, the induced fields may be able to be treated as quasi-static fields [8]. According to Faraday's law, the electric field in a sample is generated with the change of magnetic field and the corresponding equations can be written as follows

$$
\begin{gathered}
\vec{E}_{1}=-\left|\frac{\partial \vec{A}}{\partial t}\right|=-\frac{N \mu_{0}}{4 \pi} \frac{\partial I}{\partial t} \int_{C} \frac{d l}{r} . \\
\vec{E}_{2}=-\nabla \Phi . \\
\vec{E}=\vec{E}_{1}+\vec{E}_{2} . \\
\vec{J}_{e}=\sigma \vec{E} .
\end{gathered}
$$

where $\vec{E}_{1}$ is the primary field, $\vec{E}_{2}$ the secondary electric field, $\vec{E}$ the total electric field, $\vec{J}_{e}$ the current density. $\vec{A}$ and $\Phi$ are the magnetic vector potential and electrical scalar potential, respectively, $N$ is the number of turns of the coil, $I$ is the current flowing in the coil, $d l$ is the coil element, $r$ is the distance between $d l$ and the field point, $\sigma$ is the electric conductivity of the material. 
The numerical scheme employed for computing the induced electric fields and current densities is the quasi-static finite-difference (QSFD) method. Briefly, Eq. 5 is used to integrate the vector magnetic potential $\vec{A}$ inside the body model due to the gradient coil current segments.

$$
\vec{A}(\vec{r})=\frac{\mu}{4 \pi} \iiint \frac{\vec{J}_{e}^{e}\left(\vec{r}^{\prime}\right)}{\left|\vec{r}^{\prime}-\vec{r}\right|} d^{3} \vec{r}^{\prime} .
$$

where $\overrightarrow{r^{\prime}}$ is the position vector of the gradient coil wire segment, $\vec{r}$ is the position vector of the field point and $\overrightarrow{J_{e}^{e}}\left(\overrightarrow{r^{\prime}}\right)$ is the current density flowing in the wire segment.

Then the primary electric field $\vec{E}_{1}$ inside the body model is evaluated via Eq. 1 by considering the Fourier harmonic frequency at $1 \mathrm{kHz}$.

The secondary electric field $\vec{E}_{2}$ inside the body model can be obtained via the following equation [9].

$$
\Phi(\mathrm{i}, \mathrm{j}, \mathrm{k})=\frac{\Xi-f(\vec{A}) h}{\sum_{q=0}^{1}\left(\sigma_{i+q, j, k}^{a}+\sigma_{i, j+q, k}^{a}+\sigma_{i, j, k+q}^{a}\right)}
$$

where $\Xi=\sum_{q=0}^{1}\left(\Phi_{i+q, j, k} \sigma_{i+q, j, k}^{a}+\Phi_{i, j+q, k} \sigma_{i, j+q, k}^{a}+\Phi_{i, j, k+q} \sigma_{i, j, k+q}^{a}\right)$

$$
f(\vec{A})=\sum_{q=0}^{1}\left[\left(\sigma_{(i+q, j, k)}^{a} \frac{\partial \vec{A}}{\partial t}{ }_{(i+q, j, k)}\right) \bullet \hat{n}_{x}^{q}+\left(\sigma_{(i, j+q, k)}^{a} \frac{\partial \vec{A}}{\partial t}{ }_{(i, j+q, k)}\right) \bullet \hat{n}_{x}^{q}+\left(\sigma_{(i, j, k+q)}^{a} \frac{\partial \vec{A}}{\partial t}{ }_{(i, j, k+q)}\right) \bullet \hat{n}_{z}^{q}\right]
$$

in which, the subscripts $\mathrm{i}, \mathrm{j}$ and $\mathrm{k}$ indicate the cell indices, subscript q indicates two faces ( 0 or 1$)$ in $\mathrm{x}$, $\mathrm{y}, \mathrm{z}$ directions, respectively, $\hat{n}_{x}^{q}$ is the unit vector normal to the cell faces, $h$ is the cell size and $\sigma^{a}$ is the local harmonic averaged conductivity.

\section{Results}

In this simulation, the human body is studied at a cell size of $5 \mathrm{~mm}$. Since we lay emphasis on the breast region, only a local segment Ella model of $x^{*} \mathrm{y} * \mathrm{z}=106 * 60 * 28$ voxels around the breast region has been calculated. It is assumed that the coil current is oscillating sinusoidal at $1 \mathrm{kHz}$, while the current magnitude is adjusted to generate gradient field strength of $35 \mathrm{mT} / \mathrm{m}$ [10].

The induced electric fields gradient and currents in each cell of the human body model were calculated, as shown in Fig. 2-3. The simulations indicate that these induced quantities have complicated distributions due to the spatial 3D magnetic field patterns and the electrical heterogeneity of the body. It also shows that induced values of the electric fields can be larger than perhaps expected.

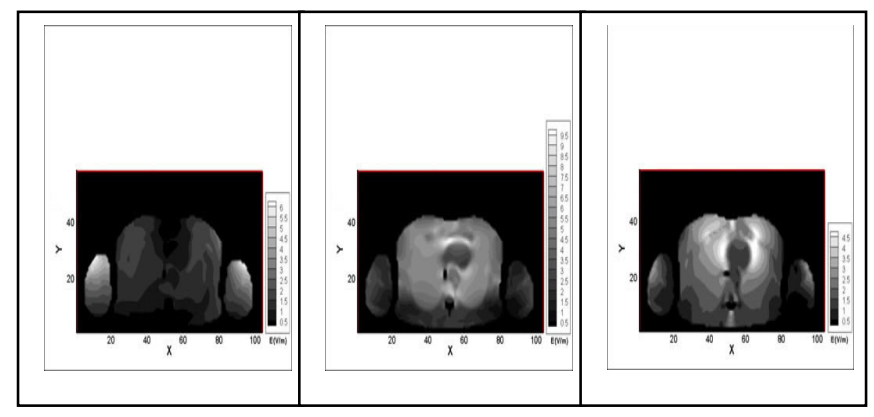




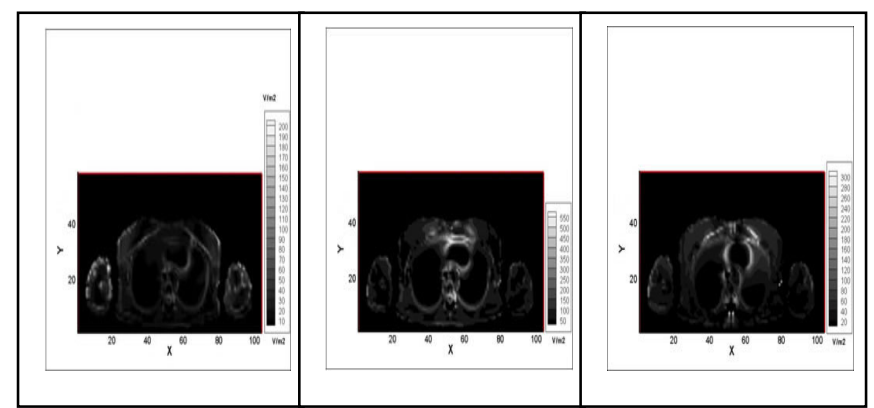

Figure 2. The distribution of the induced electric field (upper) and the electric field gradient amplitude (lower) in a transverse section XY at z=22, induced by Z coil (left), X coil (middle), and Y coil (right), respectively.

Fig. 2 illustrates the induced E-field distribution in a transverse section $X Y$ at $z=22$. It shows that the induced E-field gradient depicts the feature of induced field in the tissue more markedly than the simple E-field pattern. The E-field gradients are higher in regions of the scapula, chest, arms, oxter, heart and others which the conductivity is larger. For the $\mathrm{Z}$ coil, the larger value occurs in the arm; and for the $\mathrm{x}$ - and $\mathrm{y}$ - coils, in the chest or near the heart and the vertebra. And the E-field has the similar distribution. But the E-field gradient can show the position where the potential PNS may occur, since the regions of high conductivity or large conductivity transitions are with high risks.

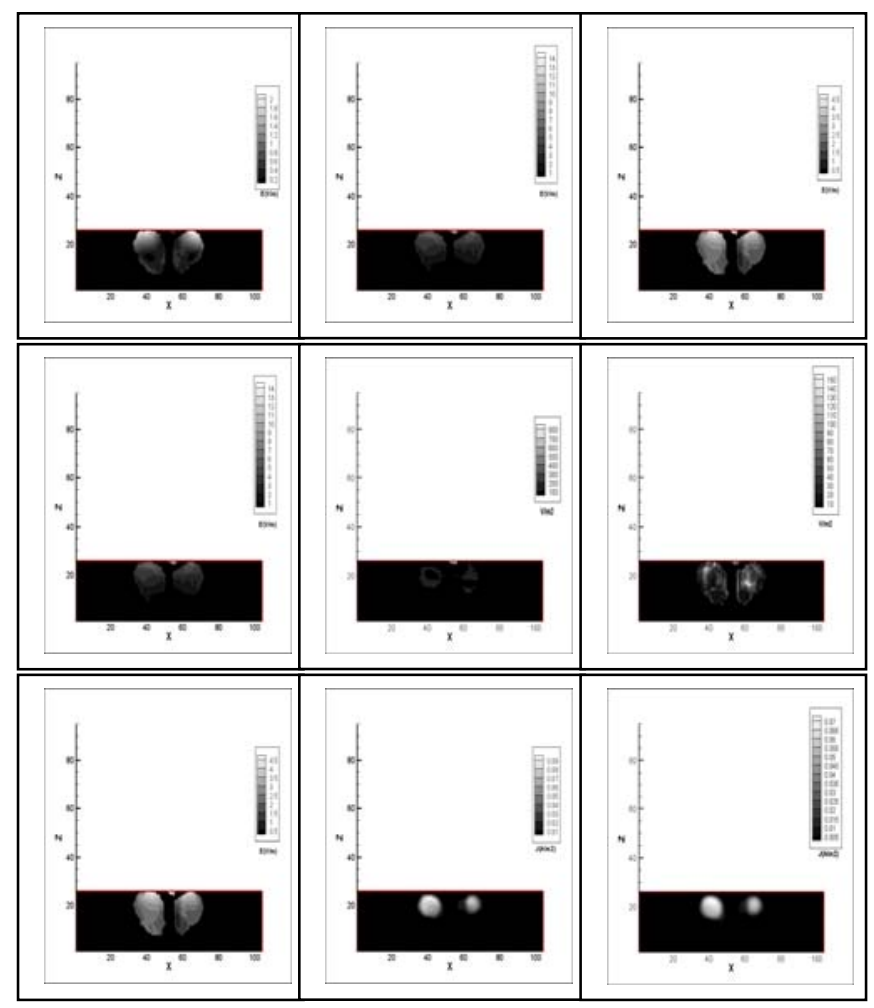

Figure 3. The distribution of the electric field (upper), the electric field gradient amplitude (middle row), and the currents densities (right) in a coronal section XZ at $\mathrm{y}=41$, induced by Z coil (left), X coil (middle), and Y coil (right), respectively.

Fig. 3 shows the induced E-field distribution in a coronal section $X Z$ at $y=41$. The larger E-field gradient value occurs in the root region (the interface of the pectoralis major and the breast fattiness) and nearly central of the breast (lobule of mammary gland region). The peak current density value appears in the root of the breast, where the interfaces between the pectoralis major and the breast fattiness, and between the fattiness and the lobule of mammary gland.

\section{Discussion and Conclusion}

In this paper, a human model of an adult female along with an efficient quasi-static finite-difference (QSFD) method was used to calculate the induced electric field/gradient distributions during MRI breast imaging. 
From the simulations, we can see that induced electric fields and currents of reasonable magnitude can be induced in patients during MRI breast tissue imaging. For the electric field, the peak value is much smaller than the threshold [11] for nerve stimulation at low frequencies, while the induced electric field gradient indicates a potential PNS [12,13].

With the recent evolution of MRI towards high-field strengths in search of improved SNR and the use of rapid imaging sequences for higher resolution, the induced electric field would fastened accordingly and could stimulate nerves and muscles, and could generate cardiac stimulation or even ventricular fibrillation [14]. These safety issues must be the object of further and properly coil designed studies. The proposed method in this paper can be used to further evaluate the sites where magnetic stimulation is likely to occur and to optimize breast gradient coil design.

\section{Reference}

[1] J. Rauschenberg, 7T \& Human Safty the Path to the Clinic Adoption, Proc. Intl. Soc. Magn. Reson. 19(2011).

[2] C. Polk and E. Postow, Eds., Handbook of Biological Effects of Electromagnetic Fields, 2nd ed., CRC, New York, 1996.

[3] C.M. Collins, S. Li, M.B. Smith, SAR and B1 field distributions in a heterogeneous human head model within a birdcage coil, Magn. Reson. Med.. 40 (1998) 847-856.

[4] T.S. Ibrahim, R. Lee, B.A. Baertlein, Y. Yu and P.M.L. Robitaille, Computational analysis of the high pass birdcage resonator: finite difference time domain solutions for high-field MRI, Magn. Reson. Imag., 18 (2000) 835-856.

[5] F. Liu, H.W. Zhao and S. Crozier, On the Induced Electric Field Gradients in the Human Body for Magnetic Stimulation by Gradient Coils in MRI IEEE Transactions on Biomedical Engineering, 50 (2003) 804-815.

[6] H.S. Lopez, M. Poole, S. Crozier, An improved equivalent magnetization current method applied to the design of local breast gradient coils, Journal of Magnetic Resonance, 199 (2009) 48-55.

[7] Information on http://niremf.ifac.cnr.it/tissprop/htmlclie/htmlclie.htm\#atsftag.

[8] D. K. Cheng, Field and wave Electromagnetics, 2nd ed. Reading, MA: Addison-Wesley, 1989.

[9] A. Trakic, Numerical Modelling of the Electromagnetic Field - Material Interactions in Magnetic Resonance Imaging, The University of Queensland, vol. The School of Information Technology and Electrical Engineering, 2007.

[10] Y. Li, J. W. Hand, T. Wills, and J. V. Hajnal, Numerically-simulated induced electric field and current density within a human model located close to a gradient coil, Journal of Magnetic Resonance Imaging, 26 (2007) 1286-1295.

[11] International Electrotechnical Commission IEC 60601-2-33 Particular requirements for basic safety and essential performance of magnetic resonance equipment for medical diagnosis, 2nd ed.; International Electrotechnical Commission: Geneva, Switzerland, 2002.

[12] W. Xi, M. A. Stuchly, and O. P. Gandhi, Induced electric currents in models of man and rodents from $60 \mathrm{~Hz}$ magnetic fields, IEEE Trans. Biomed. Eng., 41 (1994) 1018-1023.

[13] M. A. Abdeen and M. A. Stuchly, Modeling of magnetic fields stimulation of bent neurons, IEEE Trans. Biomed. Eng., 41 (1993) 1092-1095.

[14] V. Hartwig, Biological effects and safety in magnetic resonance imaging: a review, Int J Environ Res Public Health, 6 (2009) 1778-1798. 